Intelligent Traffic Control and Service in Big Data Environment, EPFL

\title{
Data-driven spatio-temporal discretization for pedestrian flow characterization
}

\author{
Marija Nikolić, Michel Bierlaire
}

August 23, 2017 


\title{
Outline
}

\author{
Introduction
}

Methodology

Application

Conclusion

TRANSP-OR 


\section{Urbanization}

1950: $30 \%$ of the population lives in cities 2014: $54 \%$ of the population lives in cities

\section{Challenges}

Energy consumption, pollution, climate change Increased traffic and congestion

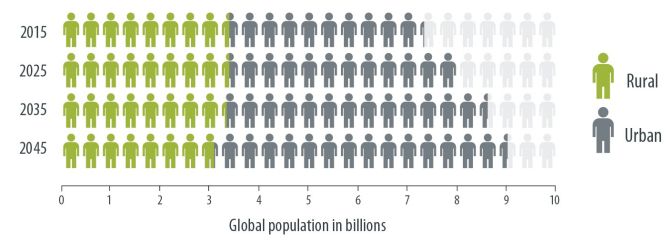




\section{Congestion: Pedestrian movements}

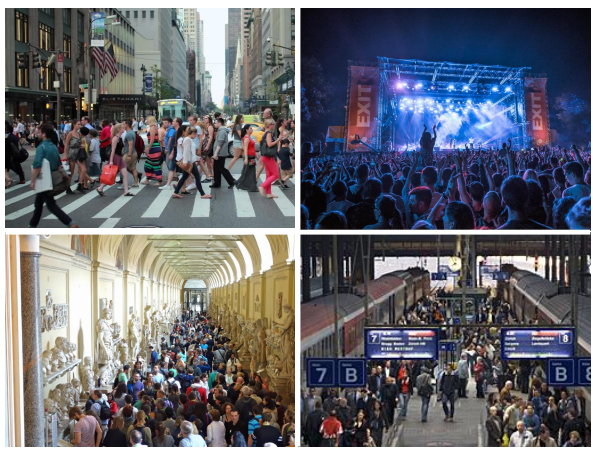

Research challenges

Understand, describe and predict

Optimization of current infrastructure and operations

Efficient planning and management of future pedestrian facilities 


\section{Characterization}

\section{Quantities}

Density $\mathrm{k}\left(\mathrm{ped} / \mathrm{m}^{2}\right)$

Speed $v(\mathrm{~m} / \mathrm{s})$

Flow q $(\mathrm{ped} / \mathrm{m} \cdot \mathrm{s})$

\section{Limitations}

Highly inspired by vehicular traffic

Arbitrary spatial and temporal discretization 


\section{Discretization}
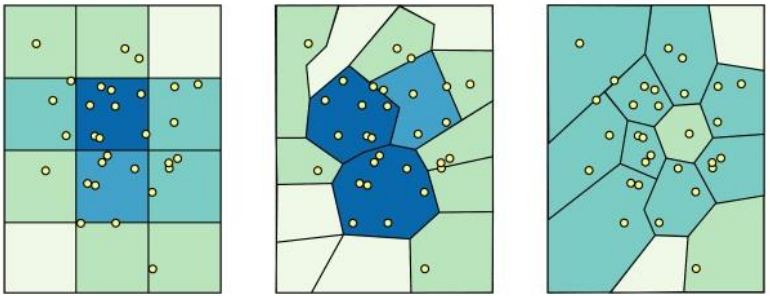

\section{Research challenges}

Results sensitive to minor changes

Arbitrary discretization may introduce noise in data 
How to define the discretization...

...independent of arbitrary chosen values?

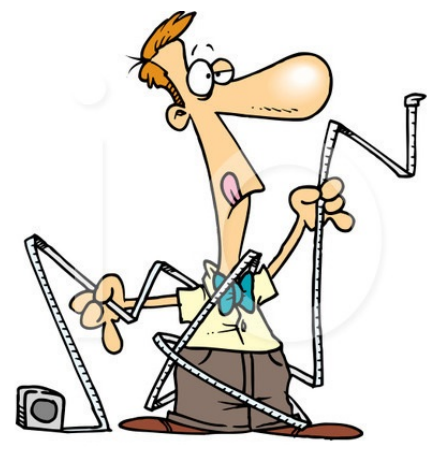

TRANSP-OR 
How to define the discretization...

...independent of arbitrary chosen values?

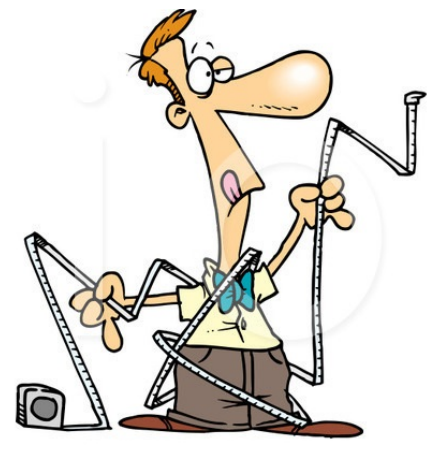

\section{Data-driven approach: Voronoi diagrams}




\section{Outline}

\section{Introduction}

Methodology

\section{Application}

Conclusion

TRANSP-OR 


\section{Context}

\section{Model}

Space-time representation: $\Omega \subset \mathbb{R}^{3}$

Units: meters and seconds

$p=(x, y, t) \in \Omega$ : physical position $(x, y)$ in space at a specific time $\mathrm{t}$

Assumption: $\Omega$ is convex (obstacle-free and bounded)

\section{Data: trajectories}

Continuous: $\Gamma_{i}:\left\{p_{i}(t) \mid p_{i}(t)=\left(x_{i}(t), y_{i}(t), t\right)\right\}$

Discrete (sample):

$\Gamma_{i}:\left\{p_{i s} \mid p_{i s}=\left(x_{i s}, y_{i s}, t_{s}\right)\right\}, t_{s}=\left[t_{0}, t_{1}, \ldots, t_{f}\right]$ 


\section{D Voronoi diagrams: 3DVoro}

\section{Definition}

Associate $p \in \Omega$ with the closest $\Gamma_{i}$ :

\section{Voronoi cell for $\Gamma_{i}$ :}

$$
\begin{gathered}
\delta_{\Gamma}\left(p, \Gamma_{i}\right)=\left\{\begin{array}{lr}
1, & D\left(p, \Gamma_{i}\right) \leq D\left(p, \Gamma_{j}\right), \forall j \\
0, & \text { otherwise }
\end{array} \quad V_{i}=\left\{p \in \mid \delta_{\Gamma}\left(p, \Gamma_{i}\right)=1\right\}\right. \\
D\left(p, \Gamma_{i}\right)=\min _{p_{i}}\left\{d\left(p, p_{i}\right)\right\}
\end{gathered}
$$

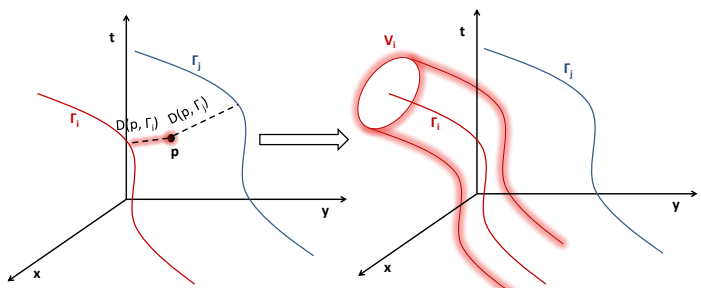




\section{DVoro: Distances}

\section{Spatial Euclidean distance}

$$
d_{E}\left(p, p_{i}\right)=\left\{\begin{array}{lr}
\sqrt{\left(x-x_{i}\right)^{2}+\left(y-y_{i}\right)^{2}}, & t=t_{i} \\
\infty, & \text { otherwise }
\end{array}\right.
$$

Each point in time is independent

Motivated by the availability of snapshots of the floor area All pedestrians must be observed at the exact same time 


\section{DVoro: Distances}

\section{Time-Transform distances}

$$
\begin{gathered}
d_{T T_{1}}\left(p, p_{i}\right)=\sqrt{\left(x-x_{i}\right)^{2}+\left(y-y_{i}\right)^{2}+v^{2}\left(t-t_{i}\right)^{2}} \\
d_{T T_{2}}\left(p, p_{i}\right)=\sqrt{\left(x-x_{i}\right)^{2}+\left(y-y_{i}\right)^{2}+\hat{v}_{i}\left(t_{i}\right)^{2}\left(t-t_{i}\right)^{2}} \\
d_{T T_{3}}\left(p, p_{i}\right)=\sqrt{\left(x-x_{i}\right)^{2}+\left(y-y_{i}\right)^{2}}+\hat{v}_{i}\left(t_{i}\right)\left|t-t_{i}\right|
\end{gathered}
$$

Convert seconds into meters using speed $d_{T T_{1}}\left(p, p_{i}\right), d_{T T_{2}}\left(p, p_{i}\right)$ : combine components based on the Euclidean norm $d_{T T_{3}}$ : weighted sum of two norms 


\section{DVoro: Distances}

\section{Predictive distance}

$$
\begin{array}{r}
d_{P}\left(p, p_{i}\right)= \begin{cases}\sqrt{\left(x_{i}^{a}-x\right)^{2}+\left(y_{i}^{a}-y\right)^{2}}, & t-t_{i} \geq 0 \\
\infty, & \text { otherwise }\end{cases} \\
x_{i}^{a}=x_{i}^{a}(t)=x_{i}+\left(t-t_{i}\right) v_{i}^{x}\left(t_{i}\right) \\
y_{i}^{a}=y_{i}^{a}(t)=y_{i}+\left(t-t_{i}\right) v_{i}^{y}\left(t_{i}\right)
\end{array}
$$

Accounts for the pedestrian dynamics Anticipates future position when performing the assignment Anticipation time: from zero to $t-t_{i}$

Points backward in time: infinitely distant 


\section{DVoro: Distances}

\section{Mahalanobis distance}

$$
d_{M}\left(p, p_{i}\right)=\sqrt{\left(p-p_{i}\right)^{T} M_{i}\left(p-p_{i}\right)}
$$

$M_{i}$ : a change of variable matrix

Points in the movement direction of a pedestrian are "closer" than the points from other directions 


\section{Intersection with a plane}

$\mathcal{P}_{(a, b, c), p_{0}}$ : plane through $p_{0}$ with normal vector $(a, b, c)$
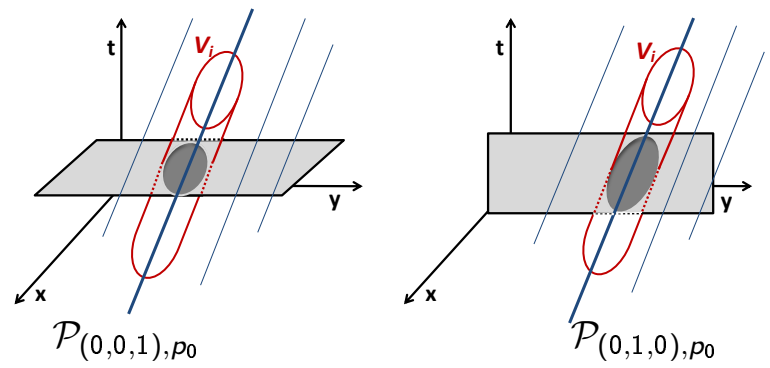


\section{Voronoi-based traffic quantities}

Consider $(x, y, t) \in \Omega$, and $i$ such that $(x, y, t) \in V_{i}$

$$
\begin{gathered}
\text { Density: } k(x, y, t)=\frac{1}{\left|V_{i} \cap \mathcal{P}_{(0,0,1),(x, y, t)}\right|} \\
\text { Flow: } \vec{q}_{(a, b, 0)}(x, y, t)=\frac{1}{\left|V_{i} \cap \mathcal{P}_{(a, b, 0),(x, y, t)}\right|} \\
\text { Velocity: } \vec{V}_{(a, b, 0)}(x, y, t)=\frac{\left|V_{i} \cap \mathcal{P}_{(0,0,1),(x, y, t)}\right|}{\left|V_{i} \cap \mathcal{P}_{(a, b, 0),(x, y, t)}\right|}
\end{gathered}
$$




\section{Outline}

\section{Introduction}

\section{Methodology}

Application

\section{Conclusion}

TRANSP-OR 


\section{Lausanne train station}

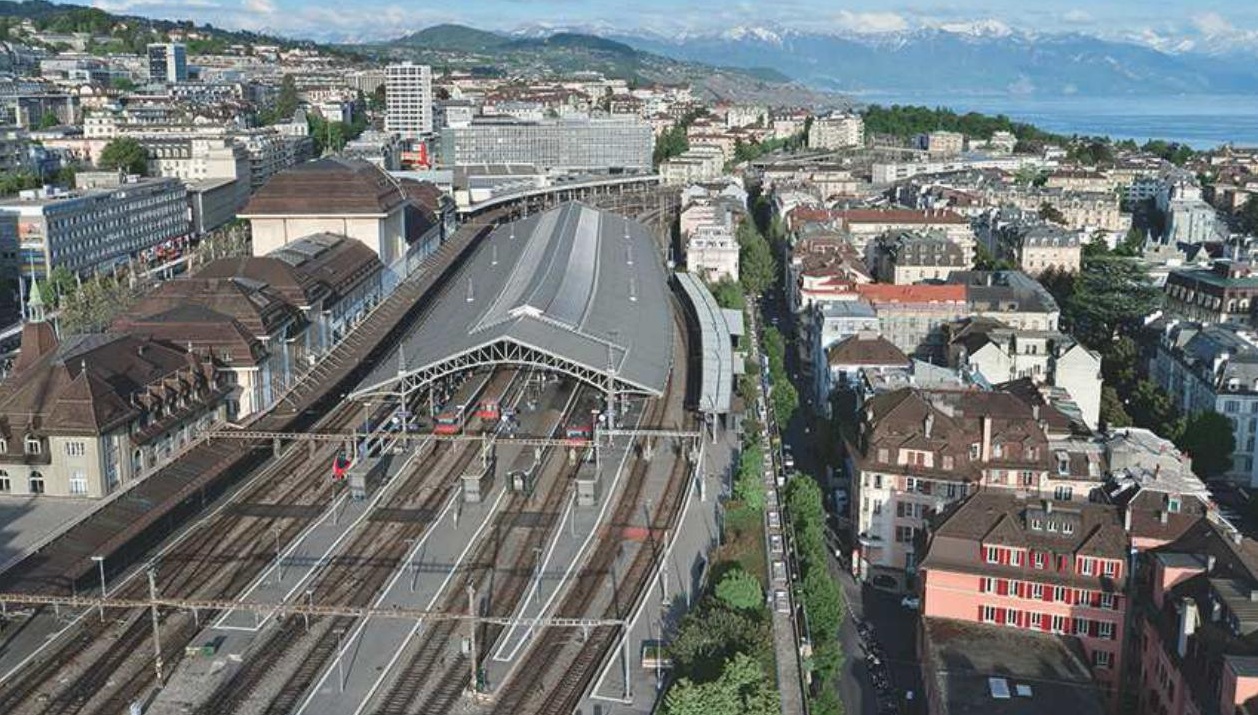




\section{Lausanne train station: Data set}

A large-scale network of smart sensors: a sparsity driven tracking (Alahi et al., 2014)

Dataset: 25,603 trajectories; February 2013

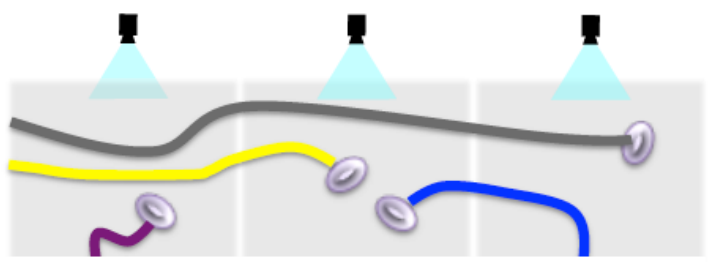




\section{DVoro illustration: Lausanne train station}
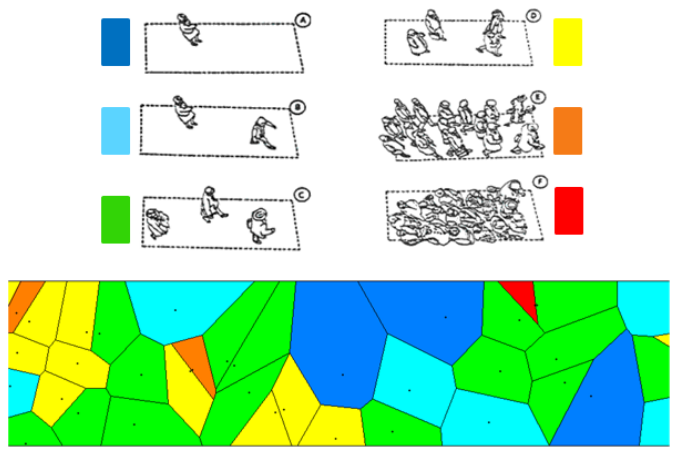

- Data-driven discretization

- General framework
- Microscopic characterization

- Applicable to continuous and discrete data 


\section{DVoro illustration: Lausanne train station}
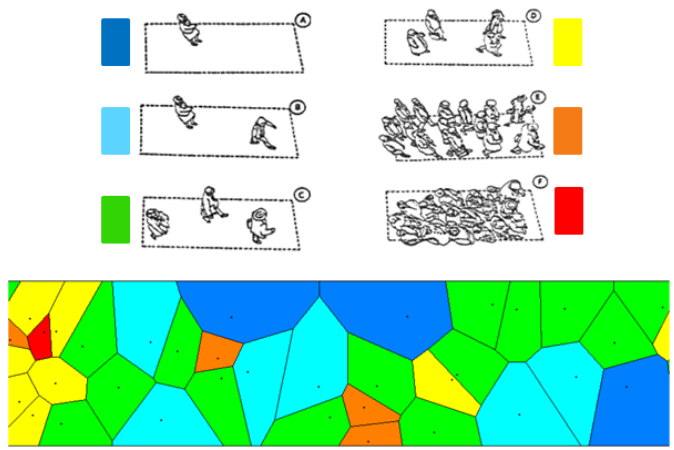

- Data-driven discretization

- General framework
- Microscopic characterization

- Applicable to continuous and discrete data 


\section{DVoro illustration: Lausanne train station}
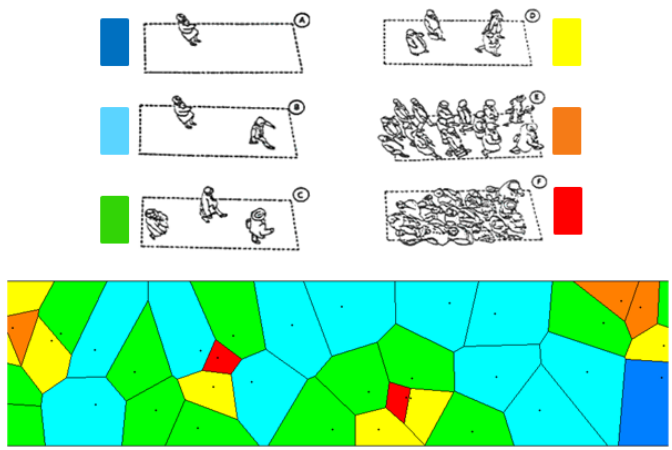

- Data-driven discretization

- General framework
- Microscopic characterization

- Applicable to continuous and discrete data 


\section{DVoro illustration: Lausanne train station}
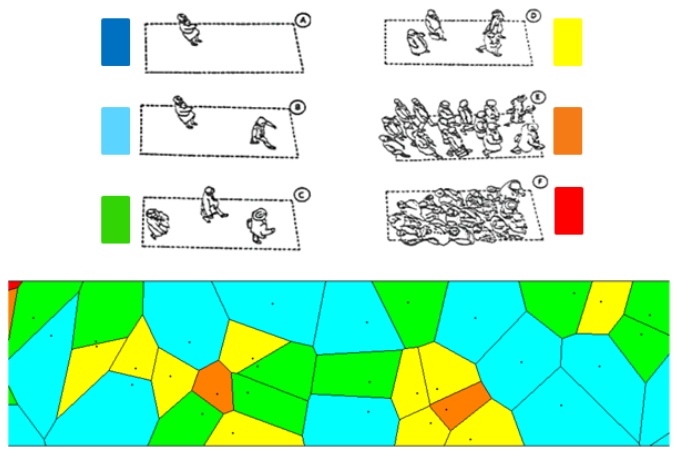

- Data-driven discretization

- General framework
- Microscopic characterization

- Applicable to continuous and discrete data 


\section{DVoro illustration: Lausanne train station}
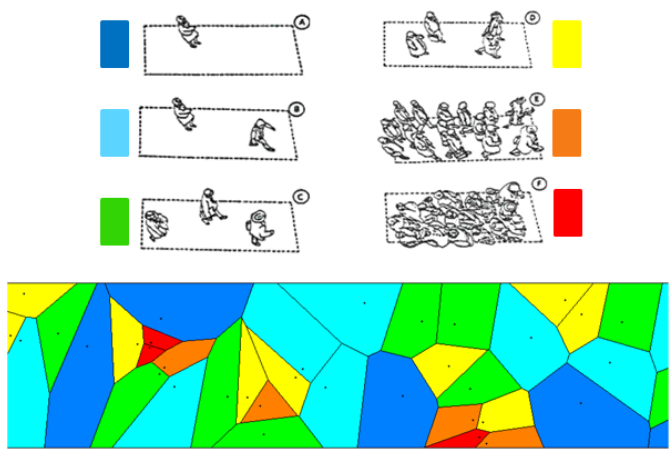

- Data-driven discretization

- General framework
- Microscopic characterization

- Applicable to continuous and discrete data 


\section{DVoro illustration: Lausanne train station}
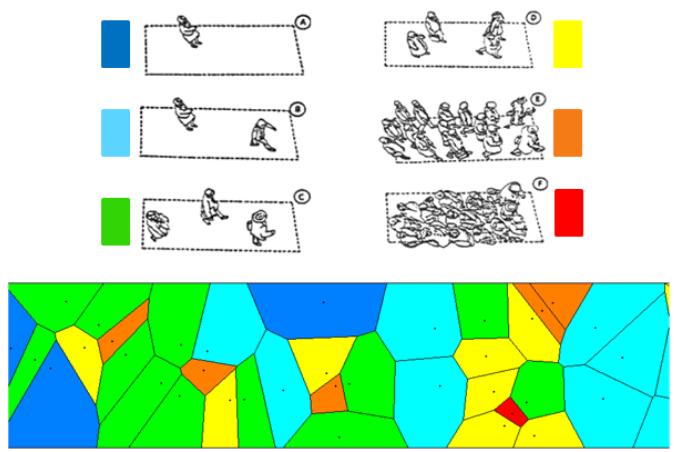

- Data-driven discretization

- General framework
- Microscopic characterization

- Applicable to continuous and discrete data 


\section{DVoro illustration: Lausanne train station}
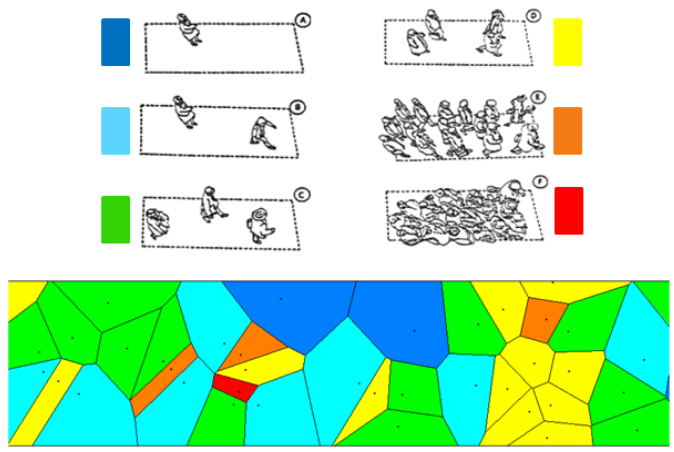

- Data-driven discretization

- General framework
- Microscopic characterization

- Applicable to continuous and discrete data 


\section{DVoro illustration: Lausanne train station}
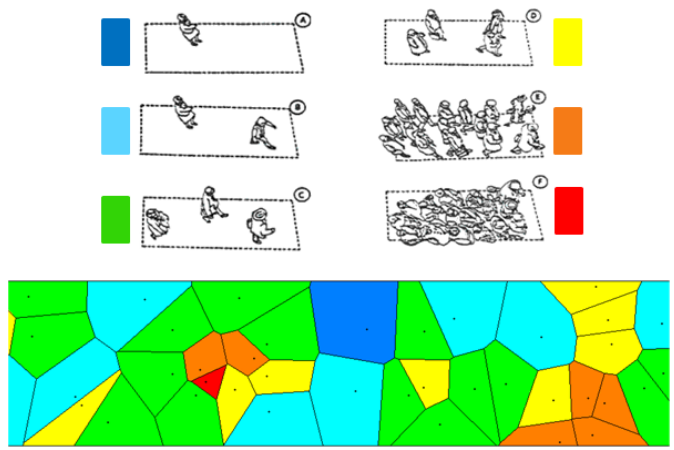

- Data-driven discretization

- General framework
- Microscopic characterization

- Applicable to continuous and discrete data 


\section{DVoro illustration: Lausanne train station}
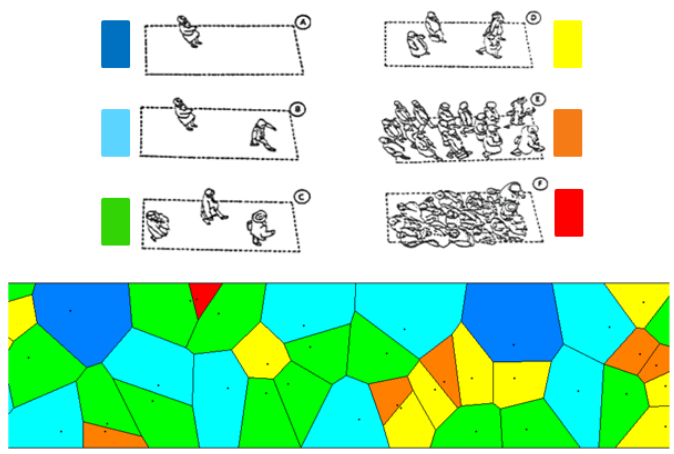

- Data-driven discretization

- General framework
- Microscopic characterization

- Applicable to continuous and discrete data 


\section{DVoro illustration: Lausanne train station}
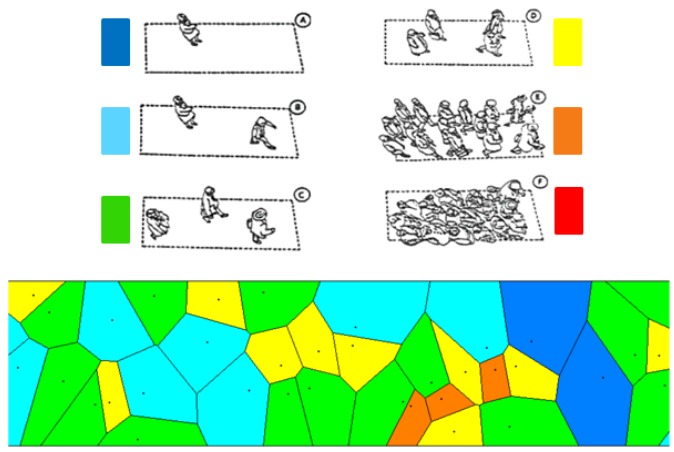

- Data-driven discretization

- General framework
- Microscopic characterization

- Applicable to continuous and discrete data 


\section{DVoro illustration: Lausanne train station}
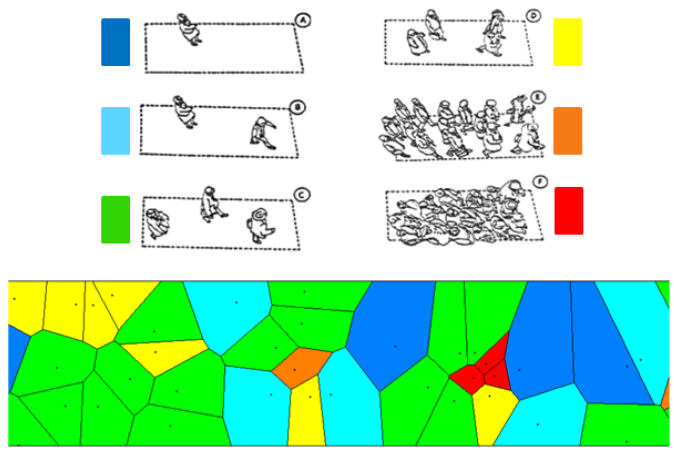

- Data-driven discretization

- General framework
- Microscopic characterization

- Applicable to continuous and discrete data 


\section{DVoro performance: Synthetic data}

NOMAD simulation tool (Campanella et al.; 2014)

Flow composition: uni-directional and bi-directional

Scenarios: low/high demand, homogenous/heterogeneous population

\section{Analysis}

3DVoro and $\mathrm{XY}-\mathrm{T}$ methods
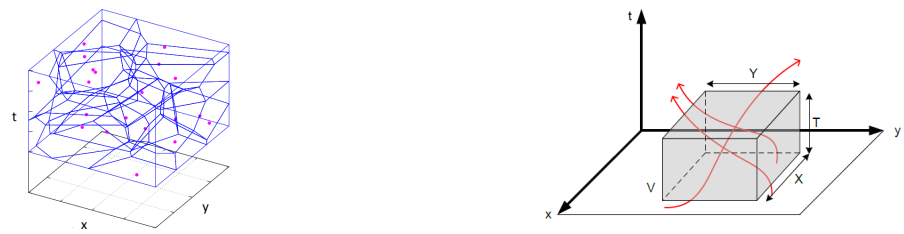


\section{Nature of the results}
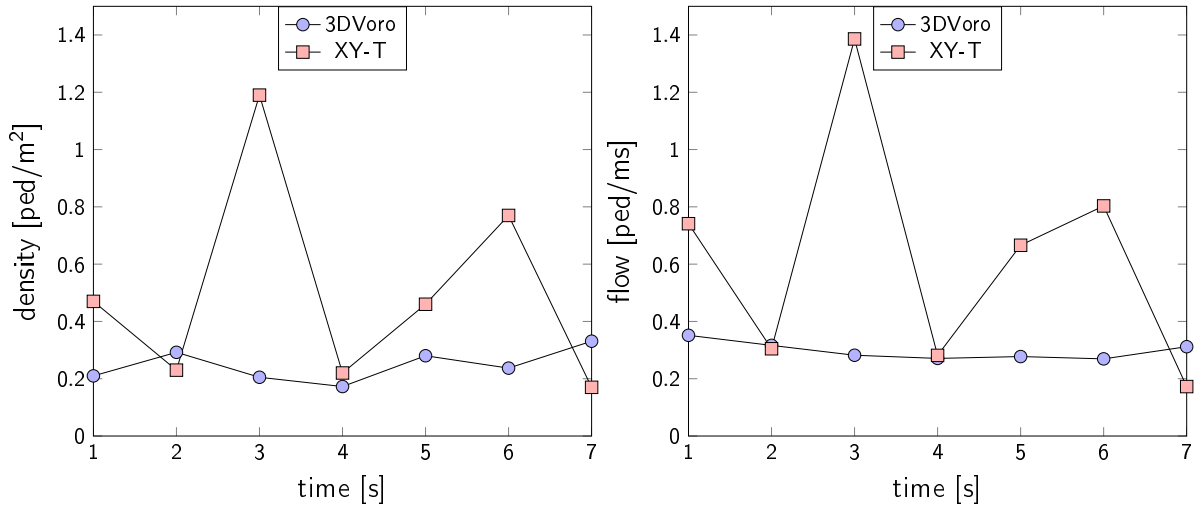


\section{Robustness to sampling of trajectories}

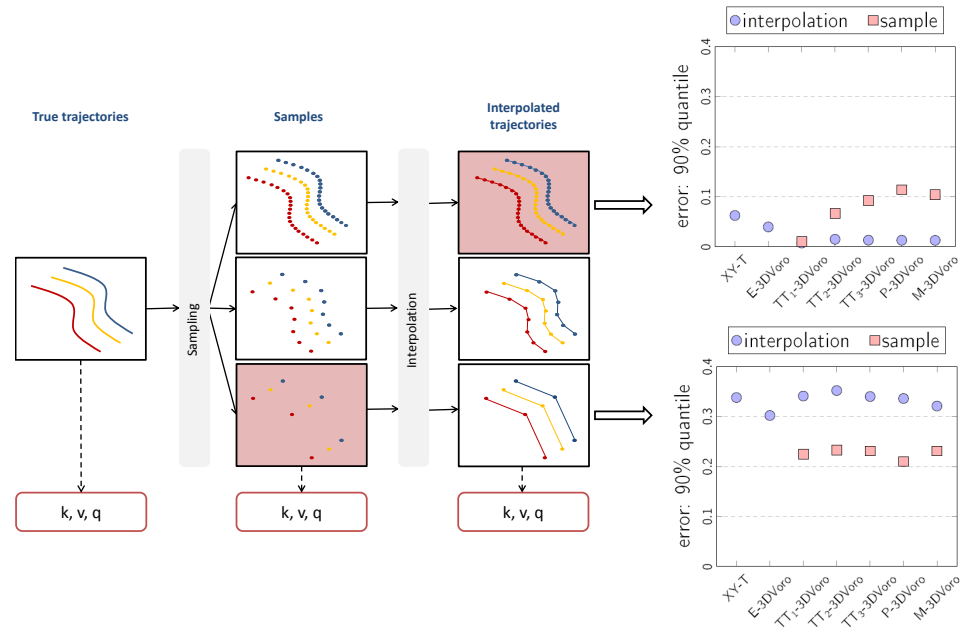




\section{Outline}

\section{Introduction}

\section{Methodology}

\section{Application}

Conclusion 


\section{Conclusion}

\section{Main findings}

Data-driven spatio-temporal discretization

Well defined, flexible and general framework

Smooth transitions in measured characteristics

Robust to noise in the data

Robust to sampling of trajectories

\section{Future directions}

Anisotropy and presence of obstacles 


\section{Thank you}

Intelligent Traffic Control and Service in Big Data Environment Data-driven spatio-temporal discretization for pedestrian flow characterization

Marija Nikolić, Michel Bierlaire

- marija.nikolic@epfl.ch 


\section{References I}

Campanella, M., Hoogendoorn, S. and Daamen, W. (2014). The nomad model: theory, developments and applications, Transportation Research Procedia 2: 462-467.

Edie, L. C. (1963). Discussion of traffic stream measurements and definitions, Port of New York Authority, New York, USA.

Fruin, J. J. (1971). Designing for pedestrians: A level-of-service concept, number 355, Highway Research Board, Washington, DC, pp. 1-15. 


\section{References II}

Helbing, D., Johansson, A. and Al-Abideen, H. Z. (2007).

Dynamics of crowd disasters: An empirical study, Physical review

E - Statistical, Nonlinear, and Soft Matter Physics 75(4): 1-7.

Jabari, S. E., Zheng, J. and Liu, H. X. (2014). A probabilistic

stationary speed-density relation based on Newell's simplified

car-following model, Transportation Research Part B:

Methodological 68: 205-223.

Saberi, M. and Mahmassani, H. (2014). Exploring areawide dynamics of pedestrian crowds: Three-dimensional approach,

Transportation Research Record: Journal of the Transportation Research Board 2421(1): 31-40. 


\section{References III}

Steffen, B. and Seyfried, A. (2010). Methods for measuring pedestrian density, flow, speed and direction with minimal scatter, Physica A: Statistical mechanics and its applications 389(9): 1902-1910.

van Wageningen-Kessels, F., Hoogendoorn, S. P. and Daamen, W. (2014). Extension of Edie's definitions for pedestrian dynamics, Transportation Research Procedia 2: 507-512. 


\section{Characteristics of methods}

\begin{tabular}{|c|c|c|c|c|c|c|}
\hline \multirow{2}{*}{ Method } & \multirow{2}{*}{ Scale } & \multicolumn{2}{|c|}{ Spatial aggregation } & \multicolumn{2}{|c|}{ Temporal aggregation } & \multirow{2}{*}{ Data type } \\
\hline & & Unit & Assumptions & Unit & Assumptions & \\
\hline$X Y-T$ & Macroscopic & Area & $\begin{array}{l}\text { Shape } \\
\text { Size } \\
\text { Location }\end{array}$ & Interval & Duration & Trajectories \\
\hline Grid-based (GB) & Macroscopic & Cell & $\begin{array}{c}\text { Size } \\
\text { Location }\end{array}$ & Interval & Duration & $\begin{array}{l}\text { Trajectories } \\
\text { Sync. sample }\end{array}$ \\
\hline Range-based (RB) & Macroscopic & Circle & $\begin{array}{l}\text { Radius } \\
\text { Location }\end{array}$ & Interval & Duration & $\begin{array}{l}\text { Trajectories } \\
\text { Sync. sample }\end{array}$ \\
\hline Exponentially-weighted (EW) & Macroscopic & Range & $\begin{array}{l}\text { Influence function } \\
\text { Range of influence }\end{array}$ & Interval & Duration & $\begin{array}{c}\text { Trajectories } \\
\text { Sync. sample }\end{array}$ \\
\hline Voronoi-based (VB) & Microscopic & Voronoi cell & Boundary conditions & Interval & Duration & $\begin{array}{l}\text { Trajectories } \\
\text { Sync. sample }\end{array}$ \\
\hline
\end{tabular}




\section{DVoro: Distances}

Mahalanobis distance

$$
\begin{array}{rc}
d^{1}\left(t_{i}\right)=\frac{v_{i}\left(t_{i}\right)}{\left\|v_{i}\left(t_{i}\right)\right\|},\left\|d^{1}\left(t_{i}\right)\right\|=1 & S_{2}\left(t_{i}, \alpha\right)=p_{i}-\Delta t v_{i}\left(t_{i}\right)-\alpha d^{1}\left(t_{i}\right) \\
d_{3}\left(t_{i}, \alpha\right)=p_{i}+\alpha d^{2}\left(t_{i}\right) \\
d^{2}\left(t_{i}\right)=\left(\begin{array}{c}
d_{x}^{1}\left(t_{i}\right) \\
d_{y}^{2}\left(t_{i}\right) \\
0
\end{array}\right) & S_{4}\left(t_{i}, \alpha\right)=p_{i}-\alpha d^{2}\left(t_{i}\right) \\
S_{5}\left(t_{i}, \alpha\right)=p_{i}+\alpha d^{3}\left(t_{i}\right) \\
d^{3}\left(t_{i}\right)=\left(\begin{array}{c}
0 \\
0 \\
\Delta t
\end{array}\right) & S_{6}\left(t_{i}, \alpha\right)=p_{i}-\alpha d^{3}\left(t_{i}\right) \\
d_{M}\left(S_{j}, p_{i}\right)=\alpha, j=1, \ldots, 6 & \mathrm{~d}^{3} s_{s_{5}\left(t_{i}, \alpha\right)}
\end{array}
$$

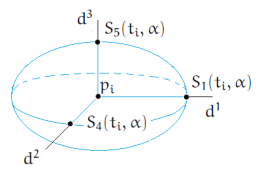


Edie (1963)

$$
\begin{array}{r}
k(A)=\frac{\sum_{i=1}^{N} t_{i}}{d x d t} \\
q(A)=\frac{\sum_{i=1}^{N} x_{i}}{d x d t} \\
v(A)=\frac{\sum_{i=1}^{N} x_{i}}{\sum_{i=1}^{N} t_{i}}
\end{array}
$$

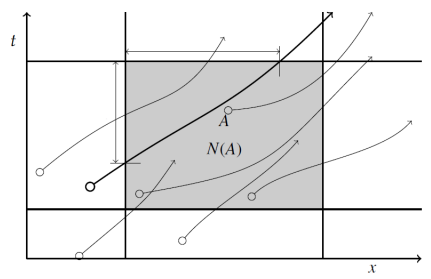


Jabari et al. (2014)

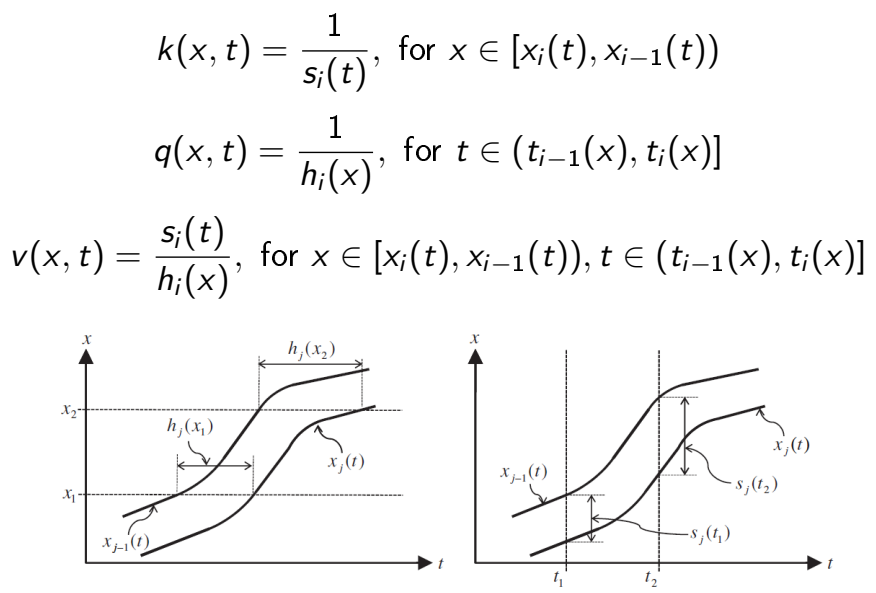




\section{Fruin (1971)}

$k(x, y, t)=\frac{N_{A}(t)}{|A|}$, for $(x, y) \in A$

$$
\vec{q}(x, y, t)=k(x, y, t) \vec{v}(x, y, t)
$$

$\vec{v}_{i}(t)=\frac{\left(\begin{array}{l}x_{i}\left(t_{2}\right) \\ y_{i}\left(t_{2}\right)\end{array}\right)-\left(\begin{array}{l}x_{i}\left(t_{1}\right) \\ y_{i}\left(t_{1}\right)\end{array}\right)}{t_{2}-t_{1}}$

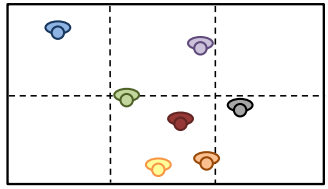

$\vec{v}(x, y, t)=\frac{\sum_{i=1}^{N_{A}} \vec{v}_{i}(t)}{N_{A}}$, for $(x, y) \in A$ 
van Wageningen-Kessels et al. (2014)

Saberi and Mahmassani (2014)

$$
k(A)=\frac{\sum_{i=1}^{N} t_{i}}{d x d y d t}
$$

$\vec{q}(A)=\left(\begin{array}{c}q_{x}(A) \\ q_{y}(A)\end{array}\right)=\left(\begin{array}{c}\sum_{i=1}^{N} x_{i} \\ \frac{d x d y d t}{N} \\ \sum_{i=1}^{N} y_{i} \\ \frac{d x d y d t}{d}\end{array}\right)$

$\vec{v}(A)=\left(\begin{array}{l}v_{x}(A) \\ v_{y}(A)\end{array}\right)=\left(\begin{array}{c}\sum_{i=1}^{N} x_{i} \\ \frac{\sum_{i=1}^{N} t_{i}}{\sum_{i=1}^{N} y_{i}} \\ \frac{\sum_{i=1}^{N} t_{i}}{2}\end{array}\right)$

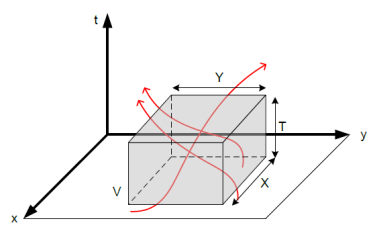




\section{Helbing et al. (2007)}

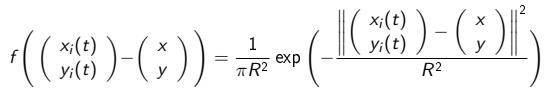

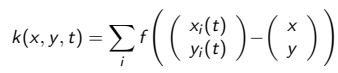

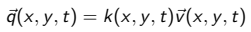

$$
\begin{aligned}
& \vec{v}_{i}(t)=\frac{\left(\begin{array}{l}
x_{i}\left(t_{2}\right) \\
y_{i}\left(t_{2}\right)
\end{array}\right)-\left(\begin{array}{l}
x_{i}\left(t_{1}\right) \\
y_{i}\left(t_{1}\right)
\end{array}\right)}{t_{2}-t_{1}} \\
& 20-1 \\
& \vec{v}(x, y, t)=\frac{\sum_{i} \vec{v}_{i}(t) f\left(\left(\begin{array}{l}
x_{i}(t) \\
y_{i}(t)
\end{array}\right)-\left(\begin{array}{l}
x \\
y
\end{array}\right)\right)}{\sum_{i} f\left(\left(\begin{array}{l}
x_{i}(t) \\
y_{i}(t)
\end{array}\right)-\left(\begin{array}{l}
x \\
y
\end{array}\right)\right)}
\end{aligned}
$$




\section{Voronoi diagrams: 2D}

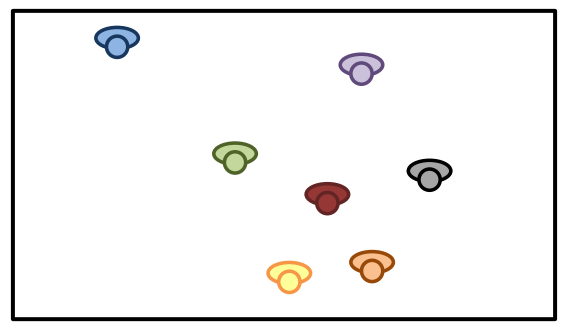




\section{Voronoi diagrams: 2D}

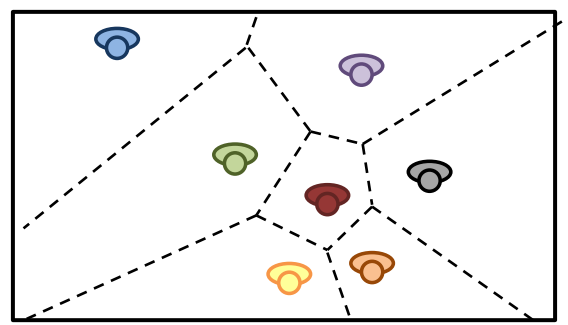


Steffen and Seyfried (2010)

$k(x, y, t)=\frac{1}{\left|A_{i}\right|}$, for $(x, y) \in A_{i}$

$\vec{v}(x, y, t)=\frac{\left(\begin{array}{l}x_{i}\left(t_{2}\right) \\ y_{i}\left(t_{2}\right)\end{array}\right)-\left(\begin{array}{l}x_{i}\left(t_{1}\right) \\ y_{i}\left(t_{1}\right)\end{array}\right)}{t_{2}-t_{1}}$

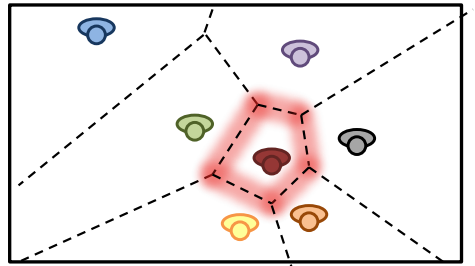

q: half a person has passed a

segment if half of the Voronoi cell

has passed it 


\section{Lausanne data}

Tracklet generation

A graph-based tracking algorithm is implemented to link the detected points

A directed graph: vertices representing the $3 \mathrm{D}$ coordinates of detected pedestrians, edges defining the connectivity between vertices The connectivity prevents too long or unrealistic connections

Tracklet association

Task: find the set of trajectories $\Theta$ that best explains the extracted tracklets

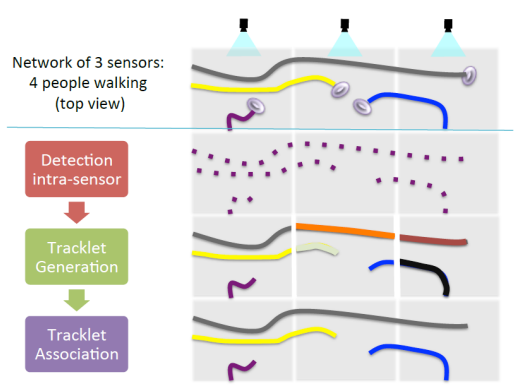

Formally:maximizing the a-posterior probability of $\Theta$ given the set of tracklets 


\section{DVoro: Robustness to noise in the data}

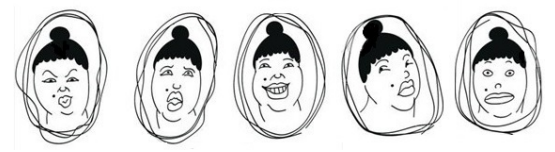

100 sets of pedestrian trajectories synthesized per scenario $\theta_{r}^{M}(p)=\left(k_{r}^{M}(p), v_{r}^{M}(p), q_{r}^{M}(p)\right)$ : a vector of indicators at point $p$ obtained by applying the method $\mathrm{M}$ to the $r^{\text {th }}$ set of trajectories

The standard deviation of the indicators at $p$ as

$$
\sigma_{R}^{M}(p)=\sqrt{\frac{1}{R} \sum_{r=1}^{R}\left(\theta_{r}^{M}(p)-\mu_{R}^{M}(p)\right)^{2}}
$$

$\mu_{R}^{M}(p)=\frac{1}{R} \sum_{r=1}^{R} \theta_{r}^{M}(p), R=100$

The procedure is repeated for 1000 randomly selected points $p$ 


\section{DVoro: Robustness to noise in the data - Sc.I}
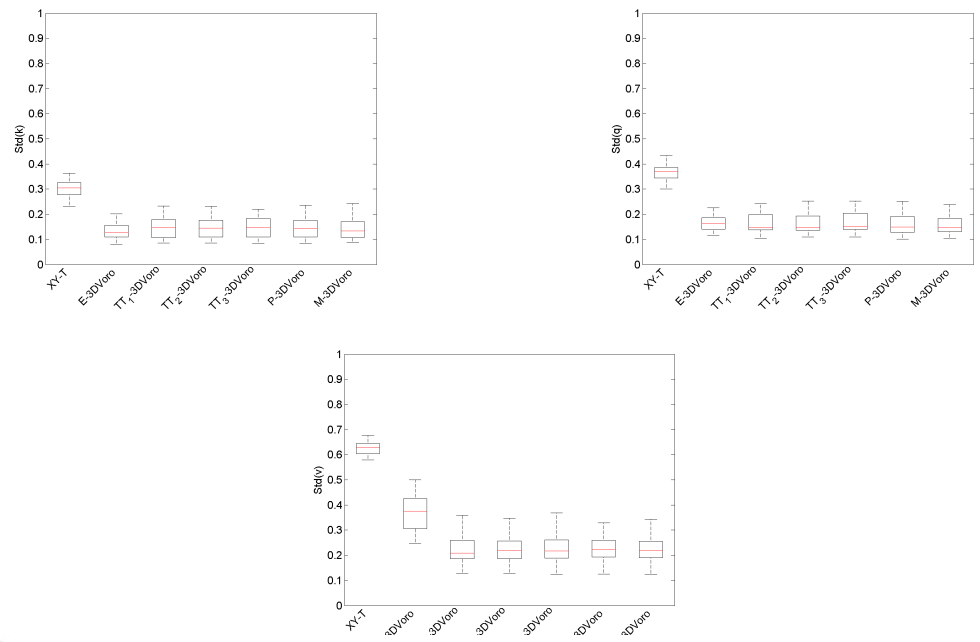


\section{DVoro: Robustness to noise in the data - Sc.II}
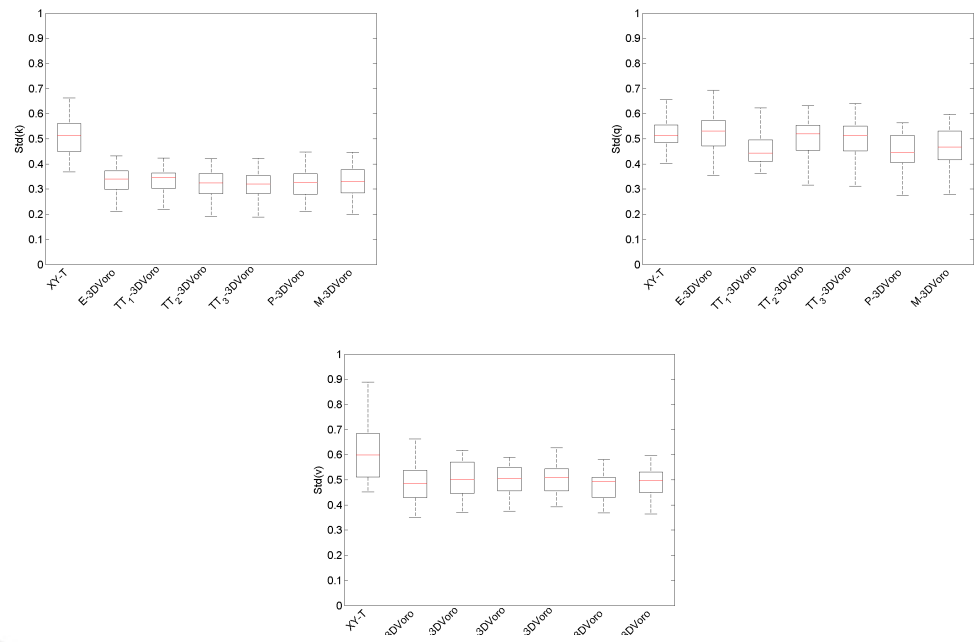

TRANSP-DR 


\section{DVoro: Robustness to sampling frequency}

\begin{tabular}{lllllllll}
\hline \multirow{2}{*}{ Method } & \multicolumn{2}{c}{ Mean } & \multicolumn{2}{c}{ Mode } & \multicolumn{2}{c}{ Median } & \multicolumn{2}{c}{$90 \%$ quantile } \\
& IT & SoP & IT & SoP & IT & SoP & IT & SoP \\
\hline XY-T & $1.47 e^{-02}$ & $/$ & $1.25 e^{-02}$ & $/$ & $1.25 e^{-02}$ & $/$ & $6.25 e^{-02}$ & $/$ \\
E-3DVoro & $1.17 e^{-02}$ & $/$ & 0 & $/$ & $4.48 e^{-04}$ & $/$ & $3.96 e^{-02}$ & $/$ \\
TT $_{1}$-3DVoro & $2.70 e^{-03}$ & $6.70 e^{-03}$ & 0 & 0 & $3.00 e^{-04}$ & $2.30 e^{-03}$ & $7.30 e^{-03}$ & $1.02 e^{-02}$ \\
TT $_{2}$-3DVoro & $5.80 e^{-03}$ & $3.50 e^{-02}$ & 0 & $2.80 e^{-03}$ & $6.00 e^{-04}$ & $2.08 e^{-02}$ & $1.50 e^{-02}$ & $6.69 e^{-02}$ \\
TT $_{3}$-3DVoro & $5.40 e^{-03}$ & $4.34 e^{-02}$ & 0 & $8.00 e^{-03}$ & $6.00 e^{-04}$ & $2.83 e^{-02}$ & $1.32 e^{-02}$ & $9.22 e^{-02}$ \\
P-3DVoro $_{\text {M-3DVoro }}$ & $8.20 e^{-03}$ & $5.36 e^{-02}$ & 0 & $6.10 e^{-03}$ & $2.40 e^{-03}$ & $3.03 e^{-02}$ & $1.30 e^{-02}$ & $1.14 e^{-01}$ \\
M-3DV & $4.50 e^{-03}$ & $5.65 e^{-02}$ & 0 & $6.80 e^{-03}$ & $1.10 e^{-03}$ & $4.55 e^{-02}$ & $1.28 e^{-02}$ & $1.04 e^{-01}$ \\
\hline
\end{tabular}

(a) Sampling frequency: $3 s^{-1}$

\begin{tabular}{|c|c|c|c|c|c|c|c|c|}
\hline \multirow{2}{*}{ Method } & \multicolumn{2}{|c|}{ Mean } & \multicolumn{2}{|c|}{ Mode } & \multicolumn{2}{|c|}{ Median } & \multicolumn{2}{|c|}{$90 \%$ quantile } \\
\hline & IT & SoP & IT & SoP & IT & SoP & IT & SoP \\
\hline$\overline{\mathrm{XY}-\mathrm{T}}$ & $1.90 e^{-01}$ & 7 & $1.00 e^{-01}$ & 7 & $1.50 e^{-01}$ & 7 & $3.38 e^{-01}$ & 7 \\
\hline E-3DVoro & $1.64 e^{-01}$ & i & $1.12 e^{-02}$ & $\bar{j}$ & $1.46 e^{-01}$ & $\%$ & $3.02 e^{-01}$ & i \\
\hline $\mathrm{TT}_{1-3 \mathrm{DV} \text { oro }}$ & $2.54 e^{-01}$ & $1.27 e^{-01}$ & $1.35 e^{-02}$ & $9.00 e^{-03}$ & $1.16 e^{-01}$ & $8.97 e^{-02}$ & $3.41 e^{-01}$ & $2.25 e^{-01}$ \\
\hline $\mathrm{TT}_{2}$-3DVoro & $1.64 e^{-01}$ & $1.22 e^{-01}$ & $1.44 e^{-02}$ & $1.06 e^{-02}$ & $1.21 e^{-01}$ & $7.30 e^{-02}$ & $3.52 e^{-01}$ & $2.33 e^{-01}$ \\
\hline $\mathrm{TT}_{3}$-3DVoro & $1.89 e^{-01}$ & $1.24 e^{-01}$ & $1.84 e^{-02}$ & $1.09 e^{-02}$ & $1.24 e^{-01}$ & $7.88 e^{-02}$ & $3.40 e^{-01}$ & $2.31 e^{-01}$ \\
\hline P-3DVoro & $3.19 e^{-01}$ & $1.21 e^{-01}$ & $3.26 e^{-02}$ & $6.20 e^{-03}$ & $1.43 e^{-01}$ & $7.43 e^{-02}$ & $3.36 e^{-01}$ & $2.10 e^{-01}$ \\
\hline M-3DVoro & $1.97 e^{-01}$ & $1.24 e^{-01}$ & $3.48 e^{-02}$ & $9.90 e^{-03}$ & $1.41 e^{-01}$ & $7.72 e^{-02}$ & $3.21 e^{-01}$ & $2.31 e^{-01}$ \\
\hline
\end{tabular}

(b) Sampling frequency: $0.5 \mathrm{~s}^{-1}$

Robustness to the sampling frequency of density indicator - Uni $i_{L D-H o m o P o p}$ 


\section{DVoro: Robustness to sampling frequency}

\begin{tabular}{|c|c|c|c|c|c|c|c|c|}
\hline \multirow{2}{*}{ Method } & \multicolumn{2}{|c|}{ Mean } & \multicolumn{2}{|c|}{ Mode } & \multicolumn{2}{|c|}{ Median } & \multicolumn{2}{|c|}{$90 \%$ quantile } \\
\hline & IT & SoP & IT & SoP & IT & SoP & IT & SoP \\
\hline$\overline{\mathrm{XY}-\mathrm{T}}$ & $2.05 e^{-02}$ & / & 0 & / & $1.25 e^{-02}$ & / & $5.00 e^{-02}$ & / \\
\hline E-3DVoro & $1.43 e^{-02}$ & i & 0 & / & $2.67 e^{-02}$ & / & $2.64 e^{-02}$ & / \\
\hline $\mathrm{TT}_{1}-3 \mathrm{DV}$ oro & $8.00 e^{-03}$ & $4,55 e^{-02}$ & 0 & 0 & $8.00 e^{-04}$ & $1.75 e^{-02}$ & $2.36 e^{-02}$ & $8.52 e^{-02}$ \\
\hline $\mathrm{TT}_{2}$-3DVoro & $1.49 e^{-02}$ & $1.07 e^{-01}$ & 0 & 0 & $3.20 e^{-03}$ & $5.72 e^{-02}$ & $3.33 e^{-02}$ & $2.21 e^{-01}$ \\
\hline $\mathrm{TT}_{3}$-3DVoro & $1.24 e^{-02}$ & $1.60 e^{-01}$ & 0 & 0 & $3.50 e^{-03}$ & $9.62 e^{-02}$ & $2.98 e^{-02}$ & $3.41 e^{-01}$ \\
\hline P-3DVoro & $2.10 e^{-02}$ & $1.66 e^{-01}$ & 0 & 0 & $4.20 e^{-03}$ & $1.16 e^{-01}$ & $5.27 e^{-02}$ & $3.64 e^{-01}$ \\
\hline M-3DVoro & $1.31 e^{-02}$ & $2.40 e^{-01}$ & 0 & 0 & $2.50 e^{-03}$ & $1.75 e^{-01}$ & $2.91 e^{-02}$ & $5.58 e^{-01}$ \\
\hline
\end{tabular}

(a) Sampling frequency: $3 s^{-1}$

\begin{tabular}{lllllllll}
\hline \multirow{2}{*}{ Method } & \multicolumn{2}{c}{ Mean } & \multicolumn{2}{c}{ Mode } & \multicolumn{2}{c}{ Median } & \multicolumn{2}{c}{$90 \%$ quantile } \\
& IT & SoP & IT & SoP & IT & SoP & IT & SoP \\
\hline XY-T & $5.29 e^{-01}$ & $/$ & $1.63 e^{-01}$ & $/$ & $4.75 e^{-01}$ & $/$ & $1.01 e^{00}$ & $/$ \\
E-3DVoro & $4.02 e^{-01}$ & $/$ & 0 & $/$ & $2.49 e^{-01}$ & $/$ & $1.03 \mathrm{E}+00$ & $/$ \\
TT $_{1}$-3DVoro & $4.06 e^{-01}$ & $2.90 e^{-01}$ & $3.10 e^{-01}$ & $2.48 e^{-02}$ & $2.64 e^{-01}$ & $1.65 e^{-01}$ & $9.21 e^{-01}$ & $7.12 e^{-01}$ \\
TT $_{2}$-3DVoro & $3.92 e^{-01}$ & $4.58 e^{-01}$ & $2.85 e^{-01}$ & $2.34 e^{-01}$ & $2.48 e^{-01}$ & $2.34 e^{-01}$ & $9.30 e^{-01}$ & $1.11 \mathrm{E}+00$ \\
TT $_{3}$-3DVoro & $4.41 e^{-01}$ & $5.07 e^{-01}$ & $2.89 e^{-01}$ & $5.89 e^{-02}$ & $2.37 e^{-01}$ & $3.06 e^{-01}$ & $9.81 e^{-01}$ & $1.17 \mathrm{E}+00$ \\
P-3DVoro & $4.31 e^{-01}$ & $3.71 e^{-01}$ & $1.40 e^{-03}$ & 0 & $2.58 e^{-01}$ & $1.80 e^{-01}$ & $9.43 e^{-01}$ & $7.29 e^{-01}$ \\
M-3DVoro & $4.34 e^{-01}$ & $5.01 e^{-01}$ & $3.16 e^{-01}$ & $1.36 e^{-01}$ & $2.75 e^{-01}$ & $3.52 e^{-01}$ & $9.96 e^{-01}$ & $9.80 e^{-01}$ \\
\hline
\end{tabular}

(b) Sampling frequency: $0.5 \mathrm{~s}^{-1}$

Robustness to the sampling frequency of density indicator - Uni $i_{H D-H e t e r o P o p}$ 


\section{DVoro: Robustness to sampling frequency}

\begin{tabular}{|c|c|c|c|c|c|c|c|c|}
\hline \multirow{2}{*}{ Method } & \multicolumn{2}{|c|}{ Mean } & \multicolumn{2}{|c|}{ Mode } & \multicolumn{2}{|c|}{ Median } & \multicolumn{2}{|c|}{$90 \%$ quantile } \\
\hline & IT & SoP & IT & SoP & IT & SoP & IT & SoP \\
\hline$\overline{X Y-T}$ & $6.50 e^{-02}$ & $/$ & 0 & $/$ & 0 & / & $8.65 e^{-03}$ & $/$ \\
\hline E-3DVoro & $1.20 e^{-02}$ & / & 0 & 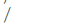 & 0 & / & $4.66 e^{-03}$ & / \\
\hline $\mathrm{TT}_{1}-3 \mathrm{DV}$ oro & $3.58 e^{-03}$ & $1.08 e^{-02}$ & 0 & 0 & 0 & $1.02 e^{-03}$ & $4.16 e^{-03}$ & $6.15 e^{-03}$ \\
\hline $\mathrm{TT}_{2} 3$ DVoro & $8.13 e^{-03}$ & $1.18 e^{-02}$ & 0 & 0 & 0 & $2.35 e^{-03}$ & $8.09 e^{-03}$ & $1.29 e^{-02}$ \\
\hline $\mathrm{TT}_{3}-3 \mathrm{DV}$ oro & $1.49 e^{-02}$ & $2.06 e^{-02}$ & 0 & $3.91 e^{-03}$ & 0 & $8.43 e^{-03}$ & $7.46 e^{-03}$ & $3.10 e^{-02}$ \\
\hline P-3DVoro & $2.29 e^{-02}$ & $5.42 e^{-02}$ & 0 & $1.94 e^{-03}$ & 0 & $2.72 e^{-02}$ & $9.25 e^{-03}$ & $1.06 e^{-01}$ \\
\hline M-3DVoro & $2.15 e^{-02}$ & $4.82 e^{-02}$ & 0 & $4.31 e^{-02}$ & 0 & $2.42 e^{-02}$ & $7.69 e^{-03}$ & $1.29 e^{-01}$ \\
\hline
\end{tabular}

(a) Sampling frequency: $3 \mathrm{~s}^{-1}$

\begin{tabular}{|c|c|c|c|c|c|c|c|c|}
\hline \multirow{2}{*}{ Method } & \multicolumn{2}{|c|}{ Mean } & \multicolumn{2}{|c|}{ Mode } & \multicolumn{2}{|c|}{ Median } & \multicolumn{2}{|c|}{$90 \%$ quantile } \\
\hline & IT & SoP & IT & SoP & IT & SoP & IT & SoP \\
\hline$\overline{X Y-T}$ & $1.66 e^{-01}$ & $/$ & 0 & / & $6.84 e^{-02}$ & / & $7.00 e^{-01}$ & / \\
\hline E-3DVoro & $1.65 e^{-01}$ & / & 0 & i & $1.19 e^{-01}$ & ; & $3.40 e^{-01}$ & / \\
\hline $\mathrm{TT}_{1}$-3DVoro & $1.68 e^{-01}$ & $1.29 e^{-01}$ & $3.50 e^{-02}$ & $5.02 e^{-02}$ & $8.50 e^{-02}$ & $5.70 e^{-02}$ & $3.85 e^{-01}$ & $2.62 e^{-01}$ \\
\hline $\mathrm{TT}_{2}-3 \mathrm{DV}$ oro & $1.70 e^{-01}$ & $1.02 e^{-01}$ & $4.52 e^{-02}$ & $5.63 e^{-02}$ & $8.49 e^{-02}$ & $6.15 e^{-02}$ & $3.82 e^{-01}$ & $5.57 e^{-01}$ \\
\hline $\mathrm{TT}_{3}$-3DVoro & $1.80 e^{-01}$ & $1.18 e^{-01}$ & $4.82 e^{-02}$ & $6.06 e^{-02}$ & $8.80 e^{-02}$ & $6.55 e^{-02}$ & $3.83 e^{-01}$ & $2.65 e^{-01}$ \\
\hline P-3DVoro & $2.02 e^{-01}$ & $1.60 e^{-01}$ & $3.69 e^{-02}$ & $4.84 e^{-02}$ & $9.36 e^{-02}$ & $6.73 e^{-02}$ & $4.14 e^{-01}$ & $3.01 e^{-01}$ \\
\hline M-3DVoro & $1.80 e^{-01}$ & $1.55 e^{-01}$ & $4.80 e^{-02}$ & $3.36 e^{-02}$ & $1.01 e^{-01}$ & $9.27 e^{-02}$ & $4.38 e^{-01}$ & $3.08 e^{-01}$ \\
\hline
\end{tabular}

(b) Sampling frequency: $0.5 \mathrm{~s}^{-1}$

Robustness to the sampling frequency of density indicator $-B i_{L D-H o m o P o p}$ 


\section{DVoro: Robustness to sampling frequency}

\begin{tabular}{|c|c|c|c|c|c|c|c|c|}
\hline \multirow{2}{*}{ Method } & \multicolumn{2}{|c|}{ Mean } & \multicolumn{2}{|r|}{ Mode } & \multicolumn{2}{|c|}{ Median } & \multicolumn{2}{|c|}{$90 \%$ quantile } \\
\hline & IT & SoP & IT & SoP & IT & SoP & IT & SoP \\
\hline$\overline{X Y-T}$ & $2.85 e^{-02}$ & $/$ & 0 & / & $3.28 e^{-03}$ & / & $1.00 e^{-01}$ & 1 \\
\hline E-3DVoro & $3.00 e^{-02}$ & ; & 0 & \% & $9.64 e^{-03}$ & \% & $6.50 e^{-02}$ & ; \\
\hline $\mathrm{TT}_{1}$-3DVoro & $1.15 e^{-01}$ & $2.78 e^{-02}$ & 0 & 0 & $7.90 e^{-04}$ & $8.78 e^{-03}$ & $2.32 e^{-02}$ & $4.94 e^{-02}$ \\
\hline $\mathrm{TT}_{2}-3 \mathrm{DV}$ oro & $9.72 e^{-02}$ & $9.34 e^{-02}$ & 0 & 0 & $3.21 e^{-03}$ & $5.16 e^{-02}$ & $3.50 e^{-02}$ & $2.15 e^{-01}$ \\
\hline $\mathrm{TT}_{3}-3 \mathrm{DV}$ oro & $4.89 e^{-02}$ & $1.05 e^{-01}$ & 0 & 0 & $2.83 e^{-03}$ & $5.91 e^{-02}$ & $3.56 e^{-02}$ & $2.62 e^{-01}$ \\
\hline P-3DVoro & $1.15 e^{-01}$ & $1.70 e^{-01}$ & 0 & $3.33 e^{-02}$ & $4.79 e^{-03}$ & $6.28 e^{-02}$ & $4.65 e^{-02}$ & $2.61 e^{-01}$ \\
\hline M-3DVoro & $1.15 e^{-01}$ & $1.52 e^{-01}$ & 0 & $8.33 e^{-02}$ & $4.55 e^{-03}$ & $7.20 e^{-02}$ & $5.35 e^{-02}$ & $3.51 e^{-01}$ \\
\hline
\end{tabular}

(a) Sampling frequency: $3 s^{-1}$

\begin{tabular}{|c|c|c|c|c|c|c|c|c|}
\hline \multirow{2}{*}{ Method } & \multicolumn{2}{|c|}{ Mean } & \multicolumn{2}{|c|}{ Mode } & \multicolumn{2}{|c|}{ Median } & \multicolumn{2}{|c|}{$90 \%$ quantile } \\
\hline & IT & SoP & IT & SoP & IT & SoP & IT & SoP \\
\hline \multicolumn{9}{|l|}{ XY-T } \\
\hline E-3DVoro & $2.79 e^{-01}$ & / & 0 & / & $1.29 e^{-01}$ & / & $7.14 e^{-01}$ & / \\
\hline $\mathrm{TT}_{1}-3 \mathrm{DV}$ oro & $4.49 e^{-01}$ & $2.58 e^{-01}$ & $5.70 e^{-03}$ & $1.99 e^{-03}$ & $1.54 e^{-01}$ & $1.34 e^{-01}$ & $8.43 e^{-01}$ & $6.64 e^{-01}$ \\
\hline $\mathrm{TT}_{2}$-3DVoro & $3.71 e^{-01}$ & $2.98 e^{-01}$ & $4.28 e^{-02}$ & $9.34 e^{-02}$ & $1.61 e^{-01}$ & $1.40 e^{-01}$ & $8.07 e^{-01}$ & $7.90 e^{-01}$ \\
\hline $\mathrm{TT}_{3}$-3DVoro & $9.82 e^{-01}$ & $3.56 e^{-01}$ & $4.34 e^{-02}$ & $6.70 e^{-03}$ & $1.64 e^{-01}$ & $1.38 e^{-01}$ & $7.76 e^{-01}$ & $7.74 e^{-01}$ \\
\hline P-3DVoro & $3.82 e^{-01}$ & $3.15 e^{-01}$ & $2.32 e^{-03}$ & $6.74 e^{-03}$ & $1.53 e^{-01}$ & $1.61 e^{-01}$ & $9.09 e^{-01}$ & $7.22 e^{-01}$ \\
\hline M-3DVoro & $4.08 e^{-01}$ & $3.77 e^{-01}$ & $1.89 e^{-02}$ & $1.47 e^{-02}$ & $1.90 e^{-01}$ & $1.74 e^{-01}$ & $7.91 e^{-01}$ & $8.18 e^{-01}$ \\
\hline
\end{tabular}

(b) Sampling frequency: $0.5 \mathrm{~s}^{-1}$

Robustness to the sampling frequency of density indicator - $B i_{H D-H e t e r o P o p}$ 


\section{DVoro: Robustness to sampling frequency}

\begin{tabular}{|c|c|c|c|c|c|c|c|c|}
\hline \multirow{2}{*}{ Method } & \multicolumn{2}{|c|}{ Mean } & \multicolumn{2}{|c|}{ Mode } & \multicolumn{2}{|c|}{ Median } & \multicolumn{2}{|c|}{$90 \%$ quantile } \\
\hline & IT & SoP & IT & SoP & IT & SoP & IT & SoP \\
\hline XY-T & $4.30 e^{-03}$ & / & 0 & $/$ & $3.40 e^{-03}$ & / & $1.16 e^{-02}$ & / \\
\hline E-3DVoro & $1.55 e^{-01}$ & / & 0 & / & $3.56 e^{-02}$ & ' & $4.99 e^{-01}$ & / \\
\hline $\mathrm{TT}_{1}-3 \mathrm{DV}$ oro & $9.60 e^{-03}$ & $2.31 e^{-02}$ & 0 & 0 & $2.20 e^{-03}$ & $9.38 e^{-03}$ & $2.79 e^{-02}$ & $4.85 e^{-02}$ \\
\hline $\mathrm{TT}_{2}-3 \mathrm{DV}$ oro & $2.04 e^{-02}$ & $7.66 e^{-02}$ & 0 & $4.10 e^{-03}$ & $5.80 e^{-03}$ & $4.48 e^{-02}$ & $6.48 e^{-02}$ & $1.68 e^{-01}$ \\
\hline $\mathrm{TT}_{3}$-3DVoro & $1.81 e^{-02}$ & $9.15 e^{-02}$ & 0 & $8.00 e^{-04}$ & $5.70 e^{-03}$ & $4.51 e^{-02}$ & $5.42 e^{-02}$ & $2.15 e^{-01}$ \\
\hline P-3DVoro & $2.98 e^{-02}$ & $1.38 e^{-01}$ & 0 & $5.90 e^{-03}$ & $1.41 e^{-02}$ & $7.90 e^{-02}$ & $5.75 e^{-02}$ & $2.92 e^{-01}$ \\
\hline M-3DVoro & $1.88 e^{-02}$ & $1.46 e^{-01}$ & 0 & $2.00 e^{-04}$ & $5.90 e^{-03}$ & $1.04 e^{-01}$ & $5.95 e^{-02}$ & $3.22 e^{-01}$ \\
\hline
\end{tabular}

(a) Sampling frequency: $3 s^{-1}$

\begin{tabular}{|c|c|c|c|c|c|c|c|c|}
\hline \multirow{2}{*}{ Method } & \multicolumn{2}{|c|}{ Mean } & \multicolumn{2}{|c|}{ Mode } & \multicolumn{2}{|c|}{ Median } & \multicolumn{2}{|c|}{$90 \%$ quantile } \\
\hline & IT & SoP & IT & SoP & IT & SoP & IT & SoP \\
\hline XY-T & $5.80 e^{-01}$ & $/$ & 1.02 & / & $3.26 e^{-01}$ & / & 1.42 & / \\
\hline E-3DVoro & 1.77 & / & $4.36 e^{-02}$ & / & $7.11 e^{-01}$ & / & 1.27 & 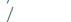 \\
\hline $\mathrm{TT}_{1}-3 \mathrm{DV}$ oro & $5.42 e^{-01}$ & $5.40 e^{-01}$ & $2.28 e^{-02}$ & $2.10 e^{-03}$ & $3.43 e^{-01}$ & $3.02 e^{-01}$ & 1.04 & $9.66 e^{-01}$ \\
\hline $\mathrm{TT}_{2}$-3DVoro & $5.11 e^{-01}$ & $5.56 e^{-01}$ & $1.39 e^{-01}$ & $8.20 e^{-03}$ & $3.15 e^{-01}$ & $3.17 e^{-01}$ & 1.07 & 1.04 \\
\hline $\mathrm{TT}_{3}$-3DVoro & $6.08 e^{-01}$ & $5.52 e^{-01}$ & $3.72 e^{-02}$ & $7.50 e^{-03}$ & $3.29 e^{-01}$ & $3.18 e^{-01}$ & 1.05 & 1.05 \\
\hline P-3DVoro & $5.60 e^{-01}$ & $5.41 e^{-01}$ & $8.75 e^{-02}$ & $1.30 e^{-03}$ & $3.32 e^{-01}$ & $3.04 e^{-01}$ & $9.76 e^{-01}$ & $9.82 e^{-01}$ \\
\hline M-3DVoro & $5.03 e^{-01}$ & $5.43 e^{-01}$ & $3.93 e^{-02}$ & $6.91 e^{-02}$ & $3.76 e^{-01}$ & $3.15 e^{-01}$ & 1.08 & $9.52 e^{-01}$ \\
\hline
\end{tabular}

(b) Sampling frequency: $0.5 \mathrm{~s}^{-1}$

Robustness to the sampling frequency of velocity indicator - Uni $L D-H o m o P o p$ 


\section{DVoro: Robustness to sampling frequency}

\begin{tabular}{lllllllll}
\hline Method & \multicolumn{2}{c}{ Mean } & \multicolumn{2}{c}{ Mode } & \multicolumn{2}{c}{ Median } & \multicolumn{2}{c}{$90 \%$ quantile } \\
& IT & SoP & IT & SoP & IT & SoP & IT & SoP \\
\hline XY-T & $1.92 e^{-02}$ & $/$ & $9.60 e^{-03}$ & $/$ & $6.20 e^{-03}$ & $/$ & $3.42 e^{-02}$ & $/$ \\
E-3DVoro & $3.17 e^{-02}$ & $/$ & 0 & $/$ & $6.30 e^{-03}$ & $/$ & $3.86 e^{-02}$ & $/$ \\
TT $_{1}$-3DVoro & $1.57 e^{-02}$ & $6.18 e^{-02}$ & 0 & 0 & $6.10 e^{-03}$ & $1.87 e^{-02}$ & $3.23 e^{-02}$ & $1.30 e^{-01}$ \\
TT $_{2}$-3DVoro & $1.83 e^{-02}$ & $1.38 e^{-01}$ & 0 & $1.73 e^{-02}$ & $7.90 e^{-03}$ & $4.27 e^{-02}$ & $3.82 e^{-02}$ & $3.88 e^{-01}$ \\
TT $_{3}$-3DVoro & $1.85 e^{-02}$ & $1.88 e^{-01}$ & 0 & $1.00 e^{-01}$ & $8.00 e^{-03}$ & $6.46 e^{-02}$ & $4.08 e^{-02}$ & $4.87 e^{-01}$ \\
P-3DVoro & $2.93 e^{-02}$ & $2.05 e^{-01}$ & 0 & $7.96 e^{-02}$ & $9.00 e^{-03}$ & $9.82 e^{-02}$ & $6.49 e^{-02}$ & $5.29 e^{-01}$ \\
M-3DVoro & $2.14 e^{-02}$ & $3.16 e^{-01}$ & 0 & $5.10 e^{-03}$ & $8.00 e^{-03}$ & $1.47 e^{-01}$ & $4.37 e^{-02}$ & $8.21 e^{-01}$ \\
\hline
\end{tabular}

(a) Sampling frequency: $3 \mathrm{~s}^{-1}$

\begin{tabular}{|c|c|c|c|c|c|c|c|c|}
\hline \multirow{2}{*}{ Method } & \multicolumn{2}{|c|}{ Mean } & \multicolumn{2}{|c|}{ Mode } & \multicolumn{2}{|c|}{ Median } & \multicolumn{2}{|c|}{$90 \%$ quantile } \\
\hline & IT & SoP & IT & SoP & IT & SoP & IT & SoP \\
\hline$\overline{X Y-T}$ & $5.73 e^{-01}$ & 7 & 1.15 & 7 & $3.51 e^{-01}$ & l & 1.58 & 7 \\
\hline E-3DVoro & 1.01 & / & $8.57 e^{-01}$ & / & $3.85 e^{-01}$ & / & 1.67 & ; \\
\hline $\mathrm{TT}_{1}-3 \mathrm{DV}$ oro & $5.82 e^{-01}$ & $5.80 e^{-01}$ & $8.69 e^{-01}$ & $5.85 e^{-02}$ & $4.51 e^{-01}$ & $3.13 e^{-01}$ & 1.40 & 1.28 \\
\hline $\mathrm{TT}_{2}-3 \mathrm{DV}$ oro & $5.76 e^{-01}$ & $5.67 e^{-01}$ & $9.40 e^{-01}$ & $1.02 e^{-01}$ & $3.75 e^{-01}$ & $2.64 e^{-01}$ & 1.54 & 1.16 \\
\hline $\mathrm{TT}_{3}-3 \mathrm{DV}$ oro & $5.79 e^{-01}$ & $5.94 e^{-01}$ & $8.50 e^{-01}$ & $5.73 e^{-02}$ & $3.70 e^{-01}$ & $2.77 e^{-01}$ & 1.46 & 1.29 \\
\hline P-3DVoro & $5.66 e^{-01}$ & $5.62 e^{-01}$ & $8.92 e^{-01}$ & $4.61 e^{-02}$ & $3.83 e^{-01}$ & $2.95 e^{-01}$ & 1.38 & 1.26 \\
\hline M-3DVoro & $6.27 e^{-01}$ & $7.11 e^{-01}$ & $9.13 e^{-01}$ & $1.43 e^{-02}$ & $5.05 e^{-01}$ & $2.86 e^{-01}$ & 1.55 & 1.49 \\
\hline
\end{tabular}

(b) Sampling frequency: $0.5 \mathrm{~s}^{-1}$

Robustness to the sampling frequency of velocity indicator - Uni $i_{H D-H e t e r o P o p}$ 


\section{DVoro: Robustness to sampling frequency}

\begin{tabular}{|c|c|c|c|c|c|c|c|c|}
\hline \multirow{2}{*}{ Method } & \multicolumn{2}{|c|}{ Mean } & \multicolumn{2}{|c|}{ Mode } & \multicolumn{2}{|c|}{ Median } & \multicolumn{2}{|c|}{$90 \%$ quantile } \\
\hline & IT & SoP & IT & SoP & IT & SoP & IT & SoP \\
\hline$\overline{\mathrm{XY}-\mathrm{T}}$ & $1.93 e^{-02}$ & / & 0 & 7 & $1.77 e^{-02}$ & / & $7.73 e^{-02}$ & 7 \\
\hline E-3DVoro & $1.65 e^{-02}$ & / & 0 & / & $5.60 e^{-03}$ & / & $3.75 e^{-02}$ & $\%$ \\
\hline $\mathrm{TT}_{1}$-3DVoro & $3.00 e^{-04}$ & $7.60 e^{-03}$ & 0 & 0 & 0 & $2.60 e^{-03}$ & $8.00 e^{-04}$ & $1.74 e^{-02}$ \\
\hline $\mathrm{TT}_{2}$-3DVoro & $1.40 e^{-03}$ & $4.16 e^{-02}$ & 0 & 0 & 0 & $3.17 e^{-02}$ & $3.60 e^{-03}$ & $8.99 e^{-02}$ \\
\hline $\mathrm{TT}_{3}$-3DVoro & $1.30 e^{-03}$ & $4.65 e^{-02}$ & 0 & $4.32 e^{-02}$ & 0 & $3.48 e^{-02}$ & $3.90 e^{-03}$ & $1.14 e^{-01}$ \\
\hline P-3DVoro & $2.70 e^{-03}$ & $4.69 e^{-02}$ & 0 & $1.41 e^{-02}$ & $8.00 e^{-04}$ & $2.27 e^{-02}$ & $5.50 e^{-03}$ & $1.29 e^{-01}$ \\
\hline M-3DVoro & $1.20 e^{-03}$ & $5.09 e^{-02}$ & 0 & $4.75 e^{-02}$ & 0 & $3.54 e^{-02}$ & $2.50 e^{-03}$ & $1.23 e^{-01}$ \\
\hline
\end{tabular}

(a) Sampling frequency: $3 s^{-1}$

\begin{tabular}{|c|c|c|c|c|c|c|c|c|}
\hline \multirow{2}{*}{ Method } & \multicolumn{2}{|c|}{ Mean } & \multicolumn{2}{|c|}{ Mode } & \multicolumn{2}{|c|}{ Median } & \multicolumn{2}{|c|}{$90 \%$ quantile } \\
\hline & IT & SoP & IT & SoP & IT & SoP & IT & SoP \\
\hline$\overline{X Y-T}$ & $2.55 e^{-01}$ & / & $1.45 e^{-01}$ & / & $2.45 e^{-01}$ & / & $5.06 e^{-01}$ & / \\
\hline E-3DVoro & $4.17 e^{-01}$ & \% & $6.50 e^{-02}$ & ; & $1.27 e^{-01}$ & / & $3.83 e^{-01}$ & \% \\
\hline 3 DVoro- $\delta_{T T_{1}}$ & $1.74 e^{-01}$ & $1.50 e^{-01}$ & $1.79 e^{-01}$ & $8.00 e^{-04}$ & $1.13 e^{-01}$ & $8.77 e^{-02}$ & $3.21 e^{-01}$ & $2.98 e^{-01}$ \\
\hline $\mathrm{TT}_{1}$-3DVoro & $2.07 e^{-01}$ & $1.53 e^{-01}$ & $1.92 e^{-01}$ & $1.00 e^{-04}$ & $1.39 e^{-01}$ & $8.52 e^{-02}$ & $3.71 e^{-01}$ & $3.29 e^{-01}$ \\
\hline $\mathrm{TT}_{2}$-3DVoro & $2.33 e^{-01}$ & $1.52 e^{-01}$ & $2.05 e^{-01}$ & $3.00 e^{-04}$ & $1.48 e^{-01}$ & $8.46 e^{-02}$ & $3.63 e^{-01}$ & $3.27 e^{-01}$ \\
\hline $\mathrm{TT}_{2}$-3DVoro & $2.17 e^{-01}$ & $1.43 e^{-01}$ & $1.53 e^{-01}$ & $1.40 e^{-03}$ & $1.34 e^{-01}$ & $8.49 e^{-02}$ & $3.01 e^{-01}$ & $2.98 e^{-01}$ \\
\hline M-3DVoro & $1.75 e^{-01}$ & $1.48 e^{-01}$ & $1.83 e^{-01}$ & $1.00 e^{-04}$ & $1.36 e^{-01}$ & $9.11 e^{-02}$ & $3.43 e^{-01}$ & $3.22 e^{-01}$ \\
\hline
\end{tabular}

(b) Sampling frequency: $0.5 \mathrm{~s}^{-1}$

Robustness to the sampling frequency of flow indicator - $U n i_{L D-H o m o P o p}$ 


\section{DVoro: Robustness to sampling frequency}

\begin{tabular}{lllllllll}
\hline \multirow{2}{*}{ Method } & \multicolumn{2}{c}{ Mean } & \multicolumn{2}{c}{ Mode } & \multicolumn{2}{c}{ Median } & \multicolumn{2}{c}{$90 \%$ quantile } \\
IT & SoP & IT & SoP & IT & SoP & IT & SoP \\
\hline XY-T & $2.75 e^{-02}$ & $/$ & $2.30 e^{-03}$ & $/$ & $1.75 e^{-02}$ & $/$ & $7.21 e^{-02}$ & $/$ \\
E-3DVoro & $1.09 e^{-02}$ & $/$ & 0 & $/$ & $8.70 e^{-04}$ & $/$ & $2.83 e^{-02}$ & $/$ \\
TT $_{1}$-3DVoro & $7.80 e^{-03}$ & $6.06 e^{-02}$ & 0 & 0 & $7.00 e^{-04}$ & $1.21 e^{-02}$ & $2.22 e^{-02}$ & $1.58 e^{-01}$ \\
TT $_{2}$-3DVoro & $1.05 e^{-02}$ & $1.45 e^{-01}$ & 0 & 0 & $1.10 e^{-03}$ & $6.08 e^{-02}$ & $2.78 e^{-02}$ & $3.11 e^{-01}$ \\
TT $_{3}$-3DVoro & $1.06 e^{-02}$ & $2.03 e^{-01}$ & 0 & 0 & $1.00 e^{-03}$ & $8.27 e^{-02}$ & $2.19 e^{-02}$ & $4.64 e^{-01}$ \\
P-3DVoro $^{1.62 e^{-02}}$ & $1.95 e^{-01}$ & 0 & $4.86 e^{-02}$ & $1.80 e^{-03}$ & $8.54 e^{-02}$ & $3.70 e^{-02}$ & $4.90 e^{-01}$ \\
M-3DVoro & $1.29 e^{-02}$ & $3.06 e^{-01}$ & 0 & 0 & $1.60 e^{-03}$ & $1.48 e^{-01}$ & $2.92 e^{-02}$ & $8.95 e^{-01}$ \\
\hline
\end{tabular}

(a) Sampling frequency: $3 \mathrm{~s}^{-1}$

\begin{tabular}{|c|c|c|c|c|c|c|c|c|}
\hline \multirow{2}{*}{ Method } & \multicolumn{2}{|c|}{ Mean } & \multicolumn{2}{|c|}{ Mode } & \multicolumn{2}{|c|}{ Median } & \multicolumn{2}{|c|}{$90 \%$ quantile } \\
\hline & IT & SoP & IT & SoP & IT & SoP & IT & SoP \\
\hline$\overline{\mathrm{XY}}-\mathrm{T}$ & $5.18 e^{-01}$ & / & $3.50 e^{-01}$ & / & $4.48 e^{-01}$ & $/$ & 1.09 & / \\
\hline E-3DVoro & $6.54 e^{-01}$ & / & $3.69 e^{-01}$ & / & $2.03 e^{-01}$ & / & 1.54 & / \\
\hline $\mathrm{TT}_{1}-3 \mathrm{DV}$ oro & $4.99 e^{-01}$ & $4.02 e^{-01}$ & $1.06 e^{-01}$ & $6.49 e^{-02}$ & $3.24 e^{-01}$ & $1.81 e^{-01}$ & 1.35 & $9.43 e^{-01}$ \\
\hline $\mathrm{TT}_{2}-3 \mathrm{DV}$ oro & $5.66 e^{-01}$ & $4.16 e^{-01}$ & $1.47 e^{-01}$ & $5.55 e^{-02}$ & $2.73 e^{-01}$ & $1.73 e^{-01}$ & 1.57 & 1.21 \\
\hline $\mathrm{TT}_{3}$-3DVoro & $5.91 e^{-01}$ & $4.45 e^{-01}$ & $1.53 e^{-01}$ & $1.57 e^{-01}$ & $2.94 e^{-01}$ & $1.71 e^{-01}$ & 1.68 & 1.31 \\
\hline P-3DVoro & $4.81 e^{-01}$ & $4.28 e^{-01}$ & $5.53 e^{-02}$ & $3.98 e^{-02}$ & $2.22 e^{-01}$ & $1.89 e^{-01}$ & 1.34 & 1.12 \\
\hline M-3DVoro & $6.41 e^{-01}$ & $4.47 e^{-01}$ & $9.07 e^{-02}$ & $4.55 e^{-02}$ & $3.97 e^{-01}$ & $1.73 e^{-01}$ & 1.66 & 1.24 \\
\hline
\end{tabular}

(b) Sampling frequency: $0.5 \mathrm{~s}^{-1}$

Robustness to the sampling frequency of flow indicator - Uni $i_{H D-H e t e r o P o p}$ 


\section{DVoro: Robustness to sampling frequency}

\section{Interpolation}

Higher sampling frequency

Time-Transform distances lead to the best performance (TT1-3DVoro)

\section{Samples}

Lower sampling frequency

Uni $i_{L D-H o m o P o p}$ : the distances that take into account the speed and/or direction of pedestrians ( $\mathrm{TT}_{2}$-3DVoro, P-3DVoro and M-3DVoro)

Uni ${ }_{H D-H e t e r o P o p}$ : Time-Transform distances ( $\mathrm{TT}_{1}-3 \mathrm{DV}$ oro)

\section{General}

Time-Transform: more data available (the sampling frequency equal to $3 \mathrm{~s}^{-1}$ or the demand equal to 3.6 pedestrians per second)

Distances accounting for the dynamics: less data available (the sampling frequency equal to $0.5 \mathrm{~s}^{-1}$ and the demand equal to 1.2 pedestrians per second) 